\title{
Study of Bioefficacy and Phytotoxicity of Carfentrazone-ethyl 40\% DF in Direct Seeded Rice
}

\author{
V. V. Shinde, D. N. Jagtap*, V. G. More, M. J. Mane and A. A. Dademal
}

Agricultural Research Station, Repoli, Dr. Balasaheb Sawant Konkan Krishi Vidyapeeth, Dapoli, Maharashtra (415 712 ), India

\section{Corresponding Author}

D. N. Jagtap

e-mail: mauli296@gmail.com

\author{
Article History \\ Article ID: AR1896 \\ Received in 02 ${ }^{\text {nd }}$ September, 2018 \\ Received in revised form $23^{\text {rd }}$ November, 2018 \\ Accepted in final form $24^{\text {th }}$ November, 2018
}

\begin{abstract}
A field experiment was conducted at ARS, Repoli ( $18^{\circ} .18^{\prime}$ North latitude and $73^{\circ} .32^{\prime}$ East longitude), to study the bioefficacy and phytotoxicity of Carfentrazone-Ethyl40 \% DF in direct seeded rice during Summer (January) of 2018. The field experiment was laid out in randomised block design with 14 treatments consists of Carfentrozone ethyl 40\% DF @ $12.5 \mathrm{~g} \mathrm{ml}^{-1}$ a.i. ha ${ }^{-1}$ at 20 DAS, Carfentrozone ethyl $40 \%$ DF @ $25 \mathrm{~g} \mathrm{ml}^{-1}$ a.i. ha ${ }^{-1}$ at 20 DAS, Carfentrozone ethyl 40\% DF @ $50 \mathrm{~g} \mathrm{ml}^{-1}$ a.i.ha ${ }^{-1}$ at 20 DAS, Carfentrozone ethyl $40 \%$ DF @ $12.5 \mathrm{~g} \mathrm{ml}^{-1}$ a.i.ha-1at $30 \mathrm{DAS}^{-}$ Carfentrozone ethyl 40 per cent DF @ $25 \mathrm{~g} \mathrm{ml}^{-1}$ a.i. ha- ${ }^{-1}$ at 30 DAS, Carfentrozone ethyl 40\% DF @ $50 \mathrm{~g} \mathrm{ml}^{-1}$ a.i.ha ${ }^{-1}$ at 30 DAS, Carfentrozone ethyl 40\% DF @ $12.5 \mathrm{~g} \mathrm{ml}^{-1}$ a.i.ha ${ }^{-1}$ at 40 DAS, Carfentrozone ethyl 40\% DF @ $25 \mathrm{~g} \mathrm{ml}^{-1}$ a.i. ha-1 at 40 DAS, Carfentrozone ethyl $40 \%$ DF @ 50 $\mathrm{g} \mathrm{ml}^{-1}$ a.i. ha-1 at 40 DAS, Carfentrozone ethyl 40\% DF @ $25 \mathrm{~g} \mathrm{ml}^{-1}$ a.i. ha ${ }^{-1}$ at 30 DAS (market product), Oxyflourofen 23.5\% EC @ 150-240 a.i. $\mathrm{ha}^{-1}$ at 30 DAS, Bispyribac sodium 10\% SC @ $20 \mathrm{~g} \mathrm{ml}^{-1}$ ha $^{-1}$ at $30 \mathrm{DAS}$, Untreated control and Hand weeding at 20 DAS and 40 DAS and three replications. Application of Carfentrozone ethyl 40\% DF @ $25 \mathrm{~g} \mathrm{ml}^{-1}$ a.i. ha- ${ }^{-1}$ at 30 DAS recorded significantly higher grain and straw yield as compared to rest of the treatments under study as well as more weed control efficiency $(86.19 \%)$ at harvest. No phytotoxicity effect was observed in any treatment after testing Carfentrozone ethyl 40\% DF in direct seeded rice.
\end{abstract}

Keywords: Bioefficacy, phytotoxicity, carfentrazone-ethyl, rice

\section{Introduction}

Rice (Oryza sativa L.) is the leading cereal of the World (FAO 2018). India is the pioneer country for the cultivation of rice crop and the second largest rice producing country in the world. Among cereals, rice has been staple food for more than $60 \%$ of the world population, providing energy for about $40 \%$ of the world population where every third person on earth consumes rice every day in one form or other (Datta et al., 2002). Therefore, crop paddy (Oryza sativa L.) is an important crop which is extensively grown in tropical and subtropical regions of the world. In India rice covering 43.57 mha amounting to $104.32 \mathrm{mt}$ of production (Anonymous 2017). West Bengal ranks first in the area ( $5.46 \mathrm{mha})$ and production (15.75 Mmt) of rice (Anonymous 2017). Among the states with considerable area under rice cultivation, Punjab has the highest rice productivity $\left(3974 \mathrm{~kg} \mathrm{ha}^{-1}\right)$ in the country followed by Tamil Nadu (3918 kg ha-1) (Anonymous, 2017). Labour required for nursery raising, uprooting and transplanting of seedlings are saved to the extent of about $40 \%$ and also saving of water (up to $60 \%$ ) as nursery raising, puddling, seepage and percolation are eliminated in direct seeded rice (Pathak et al., 2011). For this, Dry direct Seeded Rice (DSR) is the technology which is water, labour and energy efficient along with eco-friendly characteristics and can be a potential alternative to CT-TPR (Kumar and Ladha, 2011). DSR is both cost and labour-saving technology and similar or even higher yields (Hayashi et al., 2007) of DSR can be obtained with good management practices. There are several reasons for its low productivity but the losses due to weeds are one of the most important. More than one third of the total loss (33\%) is caused by weeds alone (Verma et al., 2015). Weeds are most severe and widespread biological constraints to crop production in India. Weeds are responsible for heavy yield losses in paddy, to the extent of complete crop failure under severe infestation conditions. However, heavy weed infestation is the major constraint to the production of direct seeded rice.It has been estimated that high weed infestation causes grain yield losses up to 90\% (Kaur and Singh, 2015). A weed-free period for the first 25-45 DAS is required to avoid any loss in yield in dry direct-seeded rice (Chauhan and Johnson, 2011; Singh et al., 2012). Weeds by virtue of their high adaptability and faster growth dominate the crop habitat and reduce the yield potential (Singh et al., 2004). The weeds that emerge during the later periods of crop growth cycle are not suppressed by any herbicides. Therefore, it is imperative to evaluate the efficacy of suitable post-emergence herbicides in mixture for effective control of dominant and diversified 
weed flora in direct seeded rice crop. Weeds compete for light, nutrients and water from the crop. This can be lead to the simultaneous emergence of weeds and crops and less availability of efficient selective herbicides for control of weeds during initial stages of crop weed competition. Therefore, the present investigation was undertaken to study the bioefficacy and phytotoxicity of Carfentrazone-Ethyl in direct seeded rice.

\section{Materials and Methods}

A field experiment was conducted at ARS, Repoli, Maharashtra, India (18 $.18^{\prime}$ North latitude and $73^{\circ} .32^{\prime}$ East longitude), to study the bioefficacy and phytotoxicity of Carfentrazone-Ethyl $40 \%$ DF in direct seeded rice during the Summer (January) of 2018. The field experiment was laid out in randomised block design with 14 treatments and three replications. The treatment consists of $\mathrm{T}_{1}$ : Carfentrozone ethyl 40\% DF @ 12.5 $\mathrm{g} \mathrm{ml}^{-1}$ a.i. ha ${ }^{-1}$ at $20 \mathrm{DAS}, \mathrm{T}_{2}$ : Carfentrozone ethyl 40\% DF @ $25 \mathrm{~g} \mathrm{ml}^{-1}$ a.i. ha-1 at $20 \mathrm{DAS}^{-\mathrm{T}_{3}}$ : Carfentrozone ethyl 40\% DF @ $50 \mathrm{~g} \mathrm{ml}^{-1}$ a.i. per ha at $20 \mathrm{DAS}, \mathrm{T}_{4}$ : Carfentrozone ethyl $40 \%$ DF @ $12.5 \mathrm{~g} \mathrm{ml}^{-1}$ a.i. ha- ${ }^{-1}$ at 30 DAS, $\mathrm{T}_{5}$ : Carfentrozone ethyl 40\% DF @ $25 \mathrm{~g} \mathrm{ml}^{-1}$ a.i. ha ${ }^{-1}$ at 30 DAS, $\mathrm{T}_{6}$ : Carfentrozone ethyl $40 \%$ DF @ $50 \mathrm{~g} \mathrm{ml}^{-1}$ a.i. ha ${ }^{-1}$ at 30 DAS, $T_{7}$ : Carfentrozone ethyl $40 \%$ DF @ $12.5 \mathrm{~g} \mathrm{ml}^{-1}$ a.i. ha ${ }^{-1}$ at 40 DAS, $\mathrm{T}_{8}$ : Carfentrozone ethyl $40 \%$ DF @ $25 \mathrm{~g} \mathrm{ml}^{-1}$ a.i. ha ${ }^{-1}$ at 40 DAS, T $_{9}$ : Carfentrozone ethyl $40 \%$ DF @ $50 \mathrm{~g} \mathrm{ml}^{-1}$ a.i. ha ${ }^{-1}$ at 40 DAS, $\mathrm{T}_{10}$ : Carfentrozone ethyl 40\% DF @ $25 \mathrm{~g} \mathrm{ml}^{-1}$ a.i. ha ${ }^{-1}$ at $30 \mathrm{DAS}$ (market product), $\mathrm{T}_{11}$ : Oxyflourofen $23.5 \%$ EC @ 150-240 a.i. ha ${ }^{-1}$ at 30 DAS, $\mathrm{T}_{12}$ : Bispyribac sodium 10\% SC @20 g ml $\mathrm{ha}^{-1}$ at $30 \mathrm{DAS}, \mathrm{T}_{13}$ : Untreated control and $\mathrm{T}_{14}$ : Hand weeding at 20 DAS and 40 DAS. Dibbling of sprouted rice seeds (Variety: Karjat-3) by hand with distance of $20 \mathrm{~cm}$ between row carried out on puddled soil. Carfentrazone ethyl 40\% DF applied on weed foliage using Knapsack sprayer fitted with flat fan nozzle as per treatments.

\section{Results and Discussion}

\subsection{Effect on no. of weeds}

\subsubsection{Before execution of treatments}

Data regarding number of grasses, broad leaves weeds, sedges and total number of weeds are presented in Table 1. It was observed from Table 1 that number of grasses, BLW, Sedges and total number of weeds was not influenced significantly before execution of different weed control treatments.

\subsubsection{After execution of treatments at harvest}

It was observed from Table 1 that number of grasses, BLW, Sedges and total number of weeds was influenced significantly due to different weed control treatments.

Treatment hand weeding at 20 DAS and 40 DAS recorded significantly less weed count in case of grass weeds as compared to rest of treatments under study except application of Carfentrozone ethyl 40\% DF @ $50 \mathrm{~g} \mathrm{ml}^{-1}$ a.i. ha ${ }^{-1}$ at20, 30 and 40 DAS and application of Carfentrozone ethyl 40\% DF @ $25 \mathrm{~g} \mathrm{ml}^{-1}$ a.i. ha ${ }^{-1}$ at 30 DAS which was at par with treatment hand weeding at 20 and 40 DAS.

Treatment application of Carfentrozone ethyl 40\% DF @ $50 \mathrm{~g}$ $\mathrm{ml}^{-1}$ a.i. ha-1 at 40 DASrecorded significantly less weed count in case of broad leaves weeds as compared to rest of treatments under study except treatments application of Carfentrozone ethyl 40\% DF @ $50 \mathrm{~g} \mathrm{ml}^{-1}$ a.i. ha ${ }^{-1}$ at 20 and 30 DAS, application of Carfentrozone ethyl 40\% DF @ $25 \mathrm{~g} \mathrm{ml}^{-1}$ a.i. ha ${ }^{-1}$ at 40 DAS and application of Carfentrozone ethyl 40\% DF @ $12.5 \mathrm{~g} \mathrm{ml}^{-1}$ a.i. ha ${ }^{-1}$ at 40 DAS which was at par with treatment application of Carfentrozone ethyl 40\% DF @ $50 \mathrm{~g} \mathrm{ml}^{-1}$ a.i. ha ${ }^{-1}$ at 40 DAS.

Treatment application of Carfentrozone ethyl 40\% DF @ $50 \mathrm{~g} \mathrm{ml}^{-1}$ a.i. ha ${ }^{-1}$ at 30 DAS recorded significantly less weed count in case of sedges as compared to rest of treatments under study except treatments application of Carfentrozone ethyl 40\% DF @ $25 \mathrm{~g} \mathrm{ml}^{-1}$ a.i. ha ${ }^{-1}$ at 30 DAS and application of Carfentrozone ethyl 40\% DF @ $50 \mathrm{~g} \mathrm{ml}^{-1}$ a.i. ha ${ }^{-1}$ at 20 DAS which was at par with treatment Carfentrozone ethyl $40 \%$ DF @ $50 \mathrm{~g} \mathrm{ml}^{-1}$ a.i. ha ${ }^{-1}$ at 30 DAS.

Treatment application of Carfentrozone ethyl 40\% DF @ $50 \mathrm{~g}$ $\mathrm{ml}^{-1}$ a.i. ha ${ }^{-1}$ at 40 DAS recorded significantly less weed count in case of total weed count as compared to rest of treatments under study except treatments application of Carfentrozone ethyl 40\% DF @ $25 \mathrm{~g} \mathrm{ml}^{-1}$ a.i. ha- ${ }^{-1}$ at 30 DAS, application of Carfentrozone ethyl 40\% DF @ $50 \mathrm{~g} \mathrm{ml}^{-1}$ a.i. ha ${ }^{-1}$ at 40 DAS and application of Carfentrozone ethyl 40\% DF @ $50 \mathrm{~g} \mathrm{ml}^{-1}$ a.i. ha $^{-1}$ at 20 DAS which was at par with treatment Carfentrozone ethyl 40\% DF @ $50 \mathrm{~g} \mathrm{ml}^{-1}$ a.i. ha ${ }^{-1}$ at 30 DAS. These results are in conformity with Lemerele et al. (1996), Mahajan et al. (2004) and Mahajan and Chauhan (2011).

\subsection{Effect on dry matter accumulation by weeds}

\subsubsection{Before execution of treatments}

Data regarding dry matter accumulation by grasses, broad leaves weeds, sedges and total dry matter accumulation by weeds are presented in Table 2. It was observed from Table 2 that dry matter accumulation by grasses, BLW, Sedges and total dry matter accumulation by weeds was not influenced significantly before execution of different weed control treatments.

\subsubsection{After execution of treatments}

Data regarding dry matter accumulation by grasses, broad leaves weeds, sedges and total dry matter accumulation by weeds are presented in Table 2. It was observed from Table 2 that dry matter accumulation by grasses, BLW, Sedges and total dry matter accumulation by weeds was influenced significantly due to different weed control treatments.

Treatment hand weeding at 20 and 40 DAS recorded significantly less dry matter accumulation in case of grass weeds as compared to rest of treatments under study except application of Carfentrozone ethyl 40\% DF @ $50 \mathrm{~g} \mathrm{ml}^{-1}$ a.i. ha ${ }^{-1}$ at 20, 30 and 40 DAS and application of Carfentrozone ethyl $40 \%$ DF @ $25 \mathrm{~g} \mathrm{ml}^{-1}$ a.i. ha-1 at 30 DAS which was at par with treatment hand weeding at 20 and 40 DAS. 


\begin{tabular}{|c|c|c|c|c|c|c|c|c|}
\hline \multirow[t]{2}{*}{ Treatment } & $\begin{array}{l}\text { No. of } \\
\text { grasses } \\
\left(\mathrm{m}^{-2}\right)\end{array}$ & $\begin{array}{l}\text { No. of } \\
\text { BLW } \\
\left(\mathrm{m}^{-2}\right)\end{array}$ & $\begin{array}{l}\text { No. of } \\
\text { sedges } \\
\left(\mathrm{m}^{-2}\right)\end{array}$ & $\begin{array}{c}\text { Total } \\
\text { no. of } \\
\text { weeds } \\
\left(\mathrm{m}^{-2}\right)\end{array}$ & $\begin{array}{l}\text { No. of } \\
\text { grasses } \\
\left(\mathrm{m}^{-2}\right)\end{array}$ & $\begin{array}{l}\text { No. of } \\
\text { BLW } \\
\left(\mathrm{m}^{-2}\right)\end{array}$ & $\begin{array}{l}\text { No. of } \\
\text { sedges } \\
\left(\mathrm{m}^{-2}\right)\end{array}$ & $\begin{array}{l}\text { Total no. } \\
\text { of weeds } \\
\left(\mathrm{m}^{-2}\right)\end{array}$ \\
\hline & \multicolumn{4}{|c|}{ before execution of treatments } & \multicolumn{4}{|c|}{ After execution of treatments at harvest } \\
\hline $\begin{array}{l}\text { Carfentrozone ethyl } 40 \% \text { DF @ } 12.5 \\
\mathrm{~g} \mathrm{ml}^{-1} \text { a.i. ha }{ }^{-1} \text { at } 20 \text { DAS }\end{array}$ & $\begin{array}{l}12.86 \\
(3.58)\end{array}$ & $\begin{array}{l}11.47 \\
(3.38)\end{array}$ & $\begin{array}{c}2.13 \\
(1.46)\end{array}$ & $\begin{array}{l}26.46 \\
(5.14)\end{array}$ & $\begin{array}{l}38.67 \\
(6.21)\end{array}$ & $\begin{array}{l}18.33 \\
(4.28)\end{array}$ & $\begin{array}{l}21.00 \\
(4.56)\end{array}$ & $\begin{array}{l}78.00 \\
(8.83)\end{array}$ \\
\hline $\begin{array}{l}\text { Carfentrozone ethyl } 40 \% \text { DF @ } 25 \mathrm{~g} \\
\mathrm{ml}^{-1} \text { a.i. ha }{ }^{-1} \text { at } 20 \text { DAS }\end{array}$ & $\begin{array}{l}10.32 \\
(3.19)\end{array}$ & $\begin{array}{l}11.73 \\
(3.42)\end{array}$ & $\begin{array}{c}2.27 \\
(1.50)\end{array}$ & $\begin{array}{l}24.32 \\
(4.92)\end{array}$ & $\begin{array}{l}21.33 \\
(4.62)\end{array}$ & $\begin{array}{l}16.67 \\
(4.08)\end{array}$ & $\begin{array}{l}19.00 \\
(4.35)\end{array}$ & $\begin{array}{l}57.00 \\
(7.55)\end{array}$ \\
\hline $\begin{array}{l}\text { Carfentrozone ethyl } 40 \% \text { DF @ } 50 \mathrm{~g} \\
\mathrm{ml}^{-1} \text { a.i. ha }{ }^{-1} \text { at } 20 \text { DAS }\end{array}$ & $\begin{array}{l}8.89 \\
(2.98)\end{array}$ & $\begin{array}{l}12.27 \\
(3.50)\end{array}$ & $\begin{array}{c}2.13 \\
(1.46)\end{array}$ & $\begin{array}{l}23.29 \\
(4.82)\end{array}$ & $\begin{array}{l}18.00 \\
(4.23)\end{array}$ & $\begin{array}{l}12.33 \\
(3.51)\end{array}$ & $\begin{array}{l}15.33 \\
(3.91)\end{array}$ & $\begin{array}{l}45.67 \\
(6.76)\end{array}$ \\
\hline $\begin{array}{l}\text { Carfentrozone ethyl } 40 \% \text { DF @ } 12.5 \\
\mathrm{~g} \mathrm{~m}^{-1} \text { a.i. ha-1 at } 30 \text { DAS }\end{array}$ & $\begin{array}{l}12.70 \\
(3.56)\end{array}$ & $\begin{array}{l}12.27 \\
(3.49)\end{array}$ & $\begin{array}{c}1.87 \\
(1.36)\end{array}$ & $\begin{array}{l}26.83 \\
(5.18)\end{array}$ & $\begin{array}{l}32.67 \\
(5.71)\end{array}$ & $\begin{array}{l}15.33 \\
(3.91)\end{array}$ & $\begin{array}{l}17.33 \\
(4.15)\end{array}$ & $\begin{array}{l}65.33 \\
(8.07)\end{array}$ \\
\hline $\begin{array}{l}\text { Carfentrozone ethyl } 40 \% \text { DF @ } 25 \mathrm{~g} \\
\mathrm{ml}^{-1} \text { a.i. ha }{ }^{-1} \text { at } 30 \text { DAS }\end{array}$ & $\begin{array}{l}13.97 \\
(3.74)\end{array}$ & $\begin{array}{l}12.27 \\
(3.50)\end{array}$ & $\begin{array}{l}2.00 \\
(1.41)\end{array}$ & $\begin{array}{l}28.24 \\
(5.31)\end{array}$ & $\begin{array}{l}16.00 \\
(4.00)\end{array}$ & $\begin{array}{l}16.67 \\
(4.08)\end{array}$ & $\begin{array}{l}13.33 \\
(3.63)\end{array}$ & $\begin{array}{l}46.00 \\
(6.78)\end{array}$ \\
\hline $\begin{array}{l}\text { Carfentrozone ethyl } 40 \% \text { DF @ } 50 \mathrm{~g} \\
\mathrm{ml}^{-1} \text { a.i. ha }{ }^{-1} \text { at } 30 \text { DAS }\end{array}$ & $\begin{array}{l}10.95 \\
(3.31)\end{array}$ & $\begin{array}{l}11.33 \\
(3.36)\end{array}$ & $\begin{array}{l}1.87 \\
(1.36)\end{array}$ & $\begin{array}{l}24.15 \\
(4.91)\end{array}$ & $\begin{array}{l}15.67 \\
(3.95)\end{array}$ & $\begin{array}{l}14.33 \\
(3.78)\end{array}$ & $\begin{array}{l}11.67 \\
(3.41)\end{array}$ & $\begin{array}{l}41.67 \\
(6.45)\end{array}$ \\
\hline $\begin{array}{l}\text { Carfentrozone ethyl } 40 \% \text { DF @ } 12.5 \\
\mathrm{~g} \mathrm{~m}^{-1} \text { a.i. ha-1 at } 40 \text { DAS }\end{array}$ & $\begin{array}{l}13.65 \\
(3.69)\end{array}$ & $\begin{array}{l}11.33 \\
(3.36)\end{array}$ & $\begin{array}{c}2.00 \\
(1.41)\end{array}$ & $\begin{array}{l}26.98 \\
(5.19)\end{array}$ & $\begin{array}{l}24.67 \\
(4.96)\end{array}$ & $\begin{array}{l}13.00 \\
(3.58)\end{array}$ & $\begin{array}{l}22.67 \\
(4.75)\end{array}$ & $\begin{array}{l}60.33 \\
(7.76)\end{array}$ \\
\hline $\begin{array}{l}\text { Carfentrozone ethyl } 40 \% \text { DF @ } 25 \mathrm{~g} \\
\mathrm{ml}^{-1} \text { a.i. ha }{ }^{-1} \text { at } 40 \text { DAS }\end{array}$ & $\begin{array}{l}12.38 \\
(3.51)\end{array}$ & $\begin{array}{l}11.47 \\
(3.38)\end{array}$ & $\begin{array}{l}2.27 \\
(1.50)\end{array}$ & $\begin{array}{l}26.11 \\
(5.11)\end{array}$ & $\begin{array}{l}21.33 \\
(4.61)\end{array}$ & $\begin{array}{l}11.67 \\
(3.40)\end{array}$ & $\begin{array}{l}18.67 \\
(4.31)\end{array}$ & $\begin{array}{l}51.67 \\
(7.18)\end{array}$ \\
\hline $\begin{array}{l}\text { Carfentrozone ethyl } 40 \% \text { DF @ } 50 \mathrm{~g} \\
\mathrm{ml}^{-1} \text { a.i. ha }{ }^{-1} \text { at } 40 \text { DAS }\end{array}$ & $\begin{array}{l}14.21 \\
(3.77)\end{array}$ & $\begin{array}{l}11.60 \\
(3.40)\end{array}$ & $\begin{array}{l}2.27 \\
(1.50)\end{array}$ & $\begin{array}{l}28.07 \\
(5.30)\end{array}$ & $\begin{array}{l}17.67 \\
(4.17)\end{array}$ & $\begin{array}{l}11.33 \\
(3.37)\end{array}$ & $\begin{array}{l}16.67 \\
(4.08)\end{array}$ & $\begin{array}{l}45.67 \\
(6.75)\end{array}$ \\
\hline $\begin{array}{l}\text { Carfentrozone ethyl } 40 \% \text { DF @ } 25 \\
\text { g ml }^{-1} \text { a.i. ha }{ }^{-1} \text { at } 30 \text { DAS (market } \\
\text { product) }\end{array}$ & $\begin{array}{l}12.54 \\
(3.54)\end{array}$ & $\begin{array}{l}11.47 \\
(3.37)\end{array}$ & $\begin{array}{l}2.27 \\
(1.50)\end{array}$ & $\begin{array}{l}26.27 \\
(5.12)\end{array}$ & $\begin{array}{l}21.00 \\
(4.57)\end{array}$ & $\begin{array}{l}17.67 \\
(4.20)\end{array}$ & $\begin{array}{l}21.67 \\
(4.65)\end{array}$ & $\begin{array}{l}60.33 \\
(7.76)\end{array}$ \\
\hline $\begin{array}{l}\text { Oxyflourofen } 23.5 \% \text { EC @ 150-240 } \\
\text { a.i. ha } a^{-1} \text { at } 30 \text { DAS }\end{array}$ & $\begin{array}{l}12.70 \\
(3.56)\end{array}$ & $\begin{array}{l}12.00 \\
(3.46)\end{array}$ & $\begin{array}{c}2.40 \\
(1.54)\end{array}$ & $\begin{array}{l}27.10 \\
(5.20)\end{array}$ & $\begin{array}{l}26.67 \\
(5.15)\end{array}$ & $\begin{array}{l}17.33 \\
(4.16)\end{array}$ & $\begin{array}{l}20.33 \\
(4.49)\end{array}$ & $\begin{array}{l}64.33 \\
(8.02)\end{array}$ \\
\hline $\begin{array}{l}\text { Bispyribac sodium 10\% SC @ } 20 \mathrm{~g} \\
\mathrm{ml}^{-1} \mathrm{ha}^{-1} \text { at } 30 \text { DAS }\end{array}$ & $\begin{array}{l}11.43 \\
(3.36)\end{array}$ & $\begin{array}{l}11.47 \\
(3.37)\end{array}$ & $\begin{array}{l}1.87 \\
(1.36)\end{array}$ & $\begin{array}{l}24.76 \\
(4.96)\end{array}$ & $\begin{array}{l}21.33 \\
(4.60)\end{array}$ & $\begin{array}{l}18.00 \\
(4.24)\end{array}$ & $\begin{array}{l}21.33 \\
(4.60)\end{array}$ & $\begin{array}{l}60.67 \\
(7.78)\end{array}$ \\
\hline Untreated control & $\begin{array}{l}12.30 \\
(3.50)\end{array}$ & $\begin{array}{l}11.47 \\
(3.39)\end{array}$ & $\begin{array}{l}2.27 \\
(1.50)\end{array}$ & $\begin{array}{l}26.03 \\
(5.10)\end{array}$ & $\begin{array}{l}158.33 \\
(12.58)\end{array}$ & $\begin{array}{l}56.67 \\
(7.52)\end{array}$ & $\begin{array}{l}85.00 \\
(9.22)\end{array}$ & $\begin{array}{l}300.00 \\
(17.32)\end{array}$ \\
\hline Hand weeding at 20 and 40 DAS & $\begin{array}{l}10.80 \\
(3.27)\end{array}$ & $\begin{array}{l}12.13 \\
(3.48)\end{array}$ & $\begin{array}{l}2.13 \\
(1.46)\end{array}$ & $\begin{array}{l}25.06 \\
(5.00)\end{array}$ & $\begin{array}{l}15.33 \\
(3.90)\end{array}$ & $\begin{array}{l}18.67 \\
(4.32)\end{array}$ & $\begin{array}{l}15.00 \\
(3.87)\end{array}$ & $\begin{array}{l}49.00 \\
(7.00)\end{array}$ \\
\hline SEm \pm & $\begin{array}{c}- \\
(0.15)\end{array}$ & $\begin{array}{c}- \\
(0.15)\end{array}$ & $\begin{array}{c}- \\
(0.07)\end{array}$ & $\begin{array}{c}- \\
(0.13)\end{array}$ & $\begin{array}{c}- \\
(0.21)\end{array}$ & $\begin{array}{c}- \\
(0.15)\end{array}$ & $\begin{array}{c}- \\
(0.20)\end{array}$ & $\begin{array}{c}- \\
(0.17)\end{array}$ \\
\hline $\mathrm{CD}(p=0.05)$ & NS & NS & NS & NS & $\begin{array}{c}- \\
(0.57)\end{array}$ & $\begin{array}{c}- \\
(0.41)\end{array}$ & $\begin{array}{c}- \\
(0.55)\end{array}$ & $\begin{array}{c}- \\
(0.48)\end{array}$ \\
\hline
\end{tabular}

(Values in parenthesis are square root transformed values)

Treatment application of Carfentrozone ethyl 40\% DF @ $50 \mathrm{~g}$ $\mathrm{ml}^{-1}$ a.i. ha ${ }^{-1}$ at 40 DAS recorded significantly less dry matter accumulation in case of broad leaves weeds as compared to rest of treatments under study except treatments application of Carfentrozone ethyl 40\% DF @ $50 \mathrm{~g} \mathrm{ml}^{-1}$ a.i. ha ${ }^{-1}$ at 20 DAS and application of Carfentrozone ethyl 40\% DF @ $25 \mathrm{~g} \mathrm{ml}^{-1}$ a.i. ha ${ }^{-1}$ and @ $12.5 \mathrm{~g} \mathrm{ml}^{-1}$ a.i. ha ${ }^{-1}$ at 40 DAS which was at par with treatment application of Carfentrozone ethyl 40 DF @ $50 \mathrm{~g} \mathrm{ml}^{-1}$ a.i. ha ${ }^{-1}$ at 40 DAS.

Treatment application of Carfentrozone ethyl 40\% DF @ $50 \mathrm{~g} \mathrm{ml}^{-1}$ a.i. ha ${ }^{-1}$ at 30 DAS recorded significantly less dry matter accumulation in case of sedges as compared to rest 


\begin{tabular}{|c|c|c|c|c|c|c|c|c|}
\hline \multirow[t]{2}{*}{ Treatment } & \multicolumn{4}{|c|}{$\begin{array}{l}\text { Dry matter accumulation ( } \mathrm{gm} \mathrm{m}^{-2} \text { ) } \\
\text { before execution of treatments by }\end{array}$} & \multicolumn{4}{|c|}{$\begin{array}{l}\text { Dry matter accumulation ( } \mathrm{gm} \mathrm{m}^{-2} \text { ) after } \\
\text { execution of treatments at harvest by }\end{array}$} \\
\hline & Grasses & BLW & sedges & $\begin{array}{c}\text { Total } \\
\text { no. of } \\
\text { weeds }\end{array}$ & Grasses & BLW & sedges & $\begin{array}{l}\text { Total no. } \\
\text { of weeds }\end{array}$ \\
\hline $\begin{array}{l}\text { Carfentrozone ethyl 40\% DF @ } 12.5 \mathrm{~g} \\
\mathrm{ml}^{-1} \text { a.i. ha }{ }^{-1} \text { at } 20 \text { DAS }\end{array}$ & $\begin{array}{l}62.35 \\
(7.89)\end{array}$ & $\begin{array}{l}42.43 \\
(6.51)\end{array}$ & $\begin{array}{l}10.24 \\
(3.20)\end{array}$ & $\begin{array}{l}115.02 \\
(10.72)\end{array}$ & $\begin{array}{l}302.37 \\
(17.39)\end{array}$ & $\begin{array}{l}78.47 \\
(8.85)\end{array}$ & $\begin{array}{l}87.30 \\
(9.30)\end{array}$ & $\begin{array}{l}468.14 \\
(21.63)\end{array}$ \\
\hline $\begin{array}{l}\text { Carfentrozone ethyl } 40 \% \text { DF @ } 25 \mathrm{~g} \mathrm{ml}^{-1} \\
\text { a.i. ha-1 at } 20 \text { DAS }\end{array}$ & $\begin{array}{l}50.49 \\
(7.05)\end{array}$ & $\begin{array}{l}44.11 \\
(6.64)\end{array}$ & $\begin{array}{l}10.96 \\
(3.31)\end{array}$ & $\begin{array}{l}105.55 \\
(10.26)\end{array}$ & $\begin{array}{l}166.83 \\
(12.92)\end{array}$ & $\begin{array}{l}71.33 \\
(8.44)\end{array}$ & $\begin{array}{l}78.66 \\
(8.85)\end{array}$ & $\begin{array}{l}316.82 \\
(17.80)\end{array}$ \\
\hline $\begin{array}{l}\text { Carfentrozone ethyl 40\% DF @ } 50 \mathrm{~g} \mathrm{ml}^{-1} \\
\text { a.i. ha-1 at } 20 \text { DAS }\end{array}$ & $\begin{array}{l}43.13 \\
(6.56)\end{array}$ & $\begin{array}{l}45.39 \\
(6.73)\end{array}$ & $\begin{array}{l}10.00 \\
(3.16)\end{array}$ & $\begin{array}{l}98.52 \\
(9.92)\end{array}$ & $\begin{array}{l}140.76 \\
(11.84)\end{array}$ & $\begin{array}{l}52.79 \\
(7.26)\end{array}$ & $\begin{array}{l}64.03 \\
(7.98)\end{array}$ & $\begin{array}{l}257.58 \\
(16.04)\end{array}$ \\
\hline $\begin{array}{l}\text { Carfentrozone ethyl 40\% DF @ } 12.5 \mathrm{~g} \\
\mathrm{ml}^{-1} \text { a.i. ha }{ }^{-1} \text { at } 30 \text { DAS }\end{array}$ & $\begin{array}{l}61.21 \\
(7.81)\end{array}$ & $\begin{array}{l}45.39 \\
(6.710\end{array}$ & $\begin{array}{c}9.07 \\
(3.00)\end{array}$ & $\begin{array}{l}115.67 \\
(10.75)\end{array}$ & $\begin{array}{l}254.25 \\
(15.94)\end{array}$ & $\begin{array}{l}65.63 \\
(8.10)\end{array}$ & $\begin{array}{l}71.76 \\
(8.44)\end{array}$ & $\begin{array}{l}391.64 \\
(19.78)\end{array}$ \\
\hline $\begin{array}{l}\text { Carfentrozone ethyl } 40 \% \text { DF @ } 25 \mathrm{~g} \mathrm{ml}^{-1} \\
\text { a.i. ha-1 at } 30 \text { DAS }\end{array}$ & $\begin{array}{l}67.33 \\
(8.21)\end{array}$ & $\begin{array}{l}45.39 \\
(6.73)\end{array}$ & $\begin{array}{c}9.64 \\
(3.09)\end{array}$ & $\begin{array}{l}122.36 \\
(11.06)\end{array}$ & $\begin{array}{l}125.62 \\
(11.21)\end{array}$ & $\begin{array}{l}71.33 \\
(8.44)\end{array}$ & $\begin{array}{l}56.13 \\
(7.45)\end{array}$ & $\begin{array}{l}253.09 \\
(15.91)\end{array}$ \\
\hline $\begin{array}{l}\text { Carfentrozone ethyl } 40 \% \text { DF @ } 50 \mathrm{~g} \mathrm{ml}^{-1} \\
\text { a.i. ha-1 at } 30 \text { DAS }\end{array}$ & $\begin{array}{l}52.78 \\
(7.26)\end{array}$ & $\begin{array}{l}41.93 \\
(6.45)\end{array}$ & $\begin{array}{c}8.96 \\
(2.98)\end{array}$ & $\begin{array}{l}103.67 \\
(10.17)\end{array}$ & $\begin{array}{l}122.51 \\
(11.05)\end{array}$ & $\begin{array}{l}61.35 \\
(7.82)\end{array}$ & $\begin{array}{l}48.63 \\
(6.96)\end{array}$ & $\begin{array}{l}232.49 \\
(15.24)\end{array}$ \\
\hline $\begin{array}{l}\text { Carfentrozone ethyl 40\% DF @ } 12.5 \mathrm{~g} \\
\mathrm{ml}^{-1} \text { a.i. ha }{ }^{-1} \text { at } 40 \text { DAS }\end{array}$ & $\begin{array}{l}65.79 \\
(8.11)\end{array}$ & $\begin{array}{l}41.93 \\
(6.46)\end{array}$ & $\begin{array}{c}9.60 \\
(3.09)\end{array}$ & $\begin{array}{l}117.33 \\
(10.82)\end{array}$ & $\begin{array}{l}192.89 \\
(13.87)\end{array}$ & $\begin{array}{l}55.64 \\
(7.41)\end{array}$ & $\begin{array}{l}92.97 \\
(9.63)\end{array}$ & $\begin{array}{l}341.51 \\
(18.47)\end{array}$ \\
\hline $\begin{array}{l}\text { Carfentrozone ethyl 40\% DF @ } 25 \mathrm{~g} \mathrm{ml}^{-1} \\
\text { a.i. ha-1 at } 40 \text { DAS }\end{array}$ & $\begin{array}{l}59.67 \\
(7.70)\end{array}$ & $\begin{array}{l}42.60 \\
(6.51)\end{array}$ & $\begin{array}{l}10.88 \\
(3.29)\end{array}$ & $\begin{array}{l}113.15 \\
(10.64)\end{array}$ & $\begin{array}{l}166.83 \\
(12.91)\end{array}$ & $\begin{array}{l}49.93 \\
(7.04)\end{array}$ & $\begin{array}{l}76.51 \\
(8.72)\end{array}$ & $\begin{array}{l}293.27 \\
(17.12)\end{array}$ \\
\hline $\begin{array}{l}\text { Carfentrozone ethyl } 40 \% \text { DF @ } 50 \mathrm{~g} \mathrm{ml}^{-1} \\
\text { a.i. ha-1 at } 40 \text { DAS }\end{array}$ & $\begin{array}{l}68.48 \\
(8.27)\end{array}$ & $\begin{array}{l}42.01 \\
(6.47)\end{array}$ & $\begin{array}{l}11.04 \\
(3.32)\end{array}$ & $\begin{array}{l}121.53 \\
(11.02)\end{array}$ & $\begin{array}{l}138.15 \\
(11.67)\end{array}$ & $\begin{array}{l}48.51 \\
(6.96)\end{array}$ & $\begin{array}{l}69.00 \\
(8.30)\end{array}$ & $\begin{array}{l}255.66 \\
(15.96)\end{array}$ \\
\hline $\begin{array}{l}\text { Carfentrozone ethyl } 40 \% \text { DF @ } 25 \mathrm{~g} \\
\mathrm{ml}^{-1} \text { a.i. ha-1 at } 30 \text { DAS (market product) }\end{array}$ & $\begin{array}{l}60.44 \\
(7.77)\end{array}$ & $\begin{array}{l}41.99 \\
(6.46)\end{array}$ & $\begin{array}{l}10.88 \\
(3.29)\end{array}$ & $\begin{array}{l}113.31 \\
(10.64)\end{array}$ & $\begin{array}{l}164.22 \\
(12.78)\end{array}$ & $\begin{array}{l}75.61 \\
(8.70)\end{array}$ & $\begin{array}{l}89.70 \\
(9.46)\end{array}$ & $\begin{array}{l}329.53 \\
(18.14)\end{array}$ \\
\hline $\begin{array}{l}\text { Oxyflourofen } 23.5 \% \text { EC @ 150-240 a.i. } \\
\text { ha-1 at } 30 \text { DAS }\end{array}$ & $\begin{array}{l}61.21 \\
(7.82)\end{array}$ & $\begin{array}{l}44.40 \\
(6.65)\end{array}$ & $\begin{array}{l}11.28 \\
(3.25)\end{array}$ & $\begin{array}{l}116.89 \\
(10.81)\end{array}$ & $\begin{array}{l}208.53 \\
(14.40)\end{array}$ & $\begin{array}{l}74.19 \\
(8.61)\end{array}$ & $\begin{array}{l}84.18 \\
(9.14)\end{array}$ & $\begin{array}{l}366.90 \\
(19.14)\end{array}$ \\
\hline $\begin{array}{l}\text { Bispyribac sodium } 10 \% \text { SC @ } 20 \mathrm{~g} \mathrm{ml}^{-1} \\
\text { ha-1 at } 30 \text { DAS }\end{array}$ & $\begin{array}{l}54.86 \\
(7.36)\end{array}$ & $\begin{array}{l}42.43 \\
(6.49)\end{array}$ & $\begin{array}{c}8.96 \\
(2.99)\end{array}$ & $\begin{array}{l}106.25 \\
(10.28)\end{array}$ & $\begin{array}{l}168.43 \\
(12.93)\end{array}$ & $\begin{array}{l}77.04 \\
(8.77)\end{array}$ & $\begin{array}{l}88.32 \\
(9.36)\end{array}$ & $\begin{array}{l}333.79 \\
(18.24)\end{array}$ \\
\hline Untreated control & $\begin{array}{l}59.28 \\
(7.70)\end{array}$ & $\begin{array}{l}42.43 \\
(6.51)\end{array}$ & $\begin{array}{l}11.00 \\
(3.31)\end{array}$ & $\begin{array}{l}112.71 \\
(10.61)\end{array}$ & $\begin{array}{l}1238.17 \\
(35.19)\end{array}$ & $\begin{array}{l}242.53 \\
(15.56)\end{array}$ & $\begin{array}{l}351.90 \\
(18.76)\end{array}$ & $\begin{array}{c}1832.60 \\
(42.81)\end{array}$ \\
\hline Hand weeding at 20 and 40 DAS & $\begin{array}{l}52.04 \\
(7.17)\end{array}$ & $\begin{array}{l}44.89 \\
(6.69)\end{array}$ & $\begin{array}{l}10.20 \\
(3.19)\end{array}$ & $\begin{array}{l}107.13 \\
(10.34)\end{array}$ & $\begin{array}{l}119.91 \\
(10.91)\end{array}$ & $\begin{array}{l}79.89 \\
(8.94)\end{array}$ & $\begin{array}{l}61.63 \\
(7.84)\end{array}$ & $\begin{array}{l}261.43 \\
(16.16)\end{array}$ \\
\hline SEm \pm & $\begin{array}{c}- \\
(0.33)\end{array}$ & $\begin{array}{c}- \\
(0.27)\end{array}$ & $\begin{array}{c}- \\
(0.13)\end{array}$ & $\begin{array}{c}- \\
(0.17)\end{array}$ & $\begin{array}{c}- \\
(0.56)\end{array}$ & $\begin{array}{c}- \\
(0.30)\end{array}$ & $\begin{array}{c}- \\
(0.40)\end{array}$ & $\begin{array}{c}- \\
(0.45)\end{array}$ \\
\hline $\mathrm{CD}(p=0.05)$ & NS & NS & NS & NS & $\begin{array}{c}- \\
(1.56)\end{array}$ & $\begin{array}{c}- \\
(0.84)\end{array}$ & $\begin{array}{c}- \\
(1.10)\end{array}$ & $\begin{array}{c}- \\
(1.24)\end{array}$ \\
\hline
\end{tabular}

(Values in parenthesis are square root transformed values)

of treatments under study except treatments application of Carfentrozone ethyl 40\% DF @ $25 \mathrm{~g} \mathrm{ml}^{-1}$ a.i. ha ${ }^{-1}$ at 30 DAS and application of Carfentrozone ethyl 40\% DF @ $50 \mathrm{~g} \mathrm{ml}^{-1} \mathrm{a}$.i. $\mathrm{ha}^{-1}$ at 20 DAS which was at par with treatment Carfentrozone ethyl 40\% DF @ $50 \mathrm{~g} \mathrm{~m}^{-1}$ a.i. ha ${ }^{-1}$ at 30 DAS.

Treatment application of Carfentrozone ethyl 40\% DF @ 50 $\mathrm{g} \mathrm{ml}^{-1}$ a.i. ha-1 at 30 DAS recorded significantly less dry matter accumulation in case of total weeds as compared to rest of treatments under study except treatments application of Carfentrozone ethyl 40\% DF @ $25 \mathrm{~g} \mathrm{ml}^{-1}$ a.i. ha ${ }^{-1}$ at $30 \mathrm{DAS}$, application of Carfentrozone ethyl 40\% DF @ $50 \mathrm{~g} \mathrm{ml}^{-1}$ a.i. $\mathrm{ha}^{-1}$ at 40 DAS, application of Carfentrozone ethyl $40 \%$ DF @ $50 \mathrm{~g} \mathrm{ml}^{-1}$ a.i. ha ${ }^{-1}$ at 20 DAS and hand weeding at 20 and 40 DASwhich was at par with treatment Carfentrozone ethyl $40 \%$ DF @ $50 \mathrm{~g} \mathrm{ml}^{-1}$ a.i. ha ${ }^{-1}$ at 30 DAS.These results are in 
conformity with Lemerele et al. (1996), Mahajan et al. (2004) and Mahajan and Chauhan (2011).

\subsection{Effect on yield and weed control efficiency}

Data regarding yield attributing characters of rice as influenced by different treatment at harvest is presented in Table 3. It was observed from Table 3 that number of hills $\left(\mathrm{m}^{-2}\right)$ and number of effective tillers per hill was not influenced significantly due to different weed control treatments. However, weight of grain per hill $(\mathrm{g})$ and weight of grain $\left(\mathrm{m}^{-2} \mathrm{~g}\right)$ was influenced significantly due to different weed control treatments.

Treatment application of Carfentrozone ethyl 40\% DF @ 50 $\mathrm{g} \mathrm{ml}^{-1}$ a.i. ha ${ }^{-1}$ at 30 DAS recorded significantly higher weight of grains hill ${ }^{-1}$ as compared to rest of treatments under study except treatments application of Carfentrozone ethyl $40 \%$ DF @ $25 \mathrm{~g} \mathrm{ml}^{-1}$ a.i. ha ${ }^{-1}$ at 30 DAS (market produce) and hand weeding at 20 and 40 DAS which was at par with treatment Carfentrozone ethyl 40\% DF @ $50 \mathrm{~g} \mathrm{~m}^{-1}$ a.i. ha ${ }^{-1}$ at 30 DAS.

Treatment of hand weeding at 20 and 40 DAS recorded significantly higher grain weight per sq. $\mathrm{mt}$.as compared to rest of treatments under study except application of Carfentrozoneethyl 40\% DF @ $50 \mathrm{~g} \mathrm{ml}^{-1}$ a.i. ha ${ }^{-1}$ at 30 DAS, application of Carfentrozone ethyl 40\% DF @ $25 \mathrm{~g} \mathrm{ml}^{-1}$ a.i. ha- ${ }^{-1}$ at 30 DAS and application of oxyflorofen 23.5\% EC @150240 a.i. ha ${ }^{-1}$ at 30 DAS which was at par with treatment hand weeding at 20 and 40 DAS.

Data regarding grain and straw yield of rice as influenced by

Table 3: Different yield attributing characters, grain yield, straw yield and weed control efficiency at harvest as affected by different treatments

\begin{tabular}{|c|c|c|c|c|c|c|c|c|}
\hline Treatment & $\begin{array}{l}\text { No.of } \\
\text { hills } \\
\left(\mathrm{m}^{-2}\right)\end{array}$ & $\begin{array}{l}\text { No. of } \\
\text { effective } \\
\text { tillers hill }\end{array}$ & $\begin{array}{l}\text { Weight } \\
\text { of grain } \\
\text { hill }^{-1}(g)\end{array}$ & $\begin{array}{l}\text { Weight } \\
\text { of grain } \\
\left(\mathrm{m}^{-2} \mathrm{~g}\right)\end{array}$ & $\begin{array}{l}\text { Weight } \\
\text { of straw } \\
\left(\mathrm{m}^{-2} \mathrm{~g}\right)\end{array}$ & $\begin{array}{l}\text { Grain } \\
\text { yield } \\
\left(\mathrm{kg} \mathrm{ha}^{-1}\right)\end{array}$ & $\begin{array}{l}\text { Straw } \\
\text { yield } \\
\left(\mathrm{kg} \mathrm{ha}^{-1}\right)\end{array}$ & $\begin{array}{c}\text { Weed } \\
\text { control } \\
\text { efficiency } \\
(\%)\end{array}$ \\
\hline $\begin{array}{l}\text { Carfentrozone ethyl } 40 \% \text { DF @ } \\
12.5 \mathrm{~g} \mathrm{ml}^{-1} \text { a.i. ha-1 at } 20 \text { DAS }\end{array}$ & 43.67 & 4.00 & 4.11 & 177.94 & 341.01 & 1655.40 & 3410.12 & 74.44 \\
\hline $\begin{array}{l}\text { Carfentrozone ethyl 40\% DF @ } 25 \\
\text { g ml }^{-1} \text { a.i. ha }{ }^{-1} \text { at } 20 \text { DAS }\end{array}$ & 39.33 & 4.33 & 4.91 & 192.02 & 367.83 & 1785.60 & 3678.34 & 82.72 \\
\hline $\begin{array}{l}\text { Carfentrozone ethyl 40\% DF @ } 50 \\
\mathrm{~g} \mathrm{ml}^{-1} \text { a.i. ha }{ }^{-1} \text { at } 20 \text { DAS }\end{array}$ & 46.33 & 4.33 & 4.29 & 198.70 & 370.09 & 1847.60 & 3700.90 & 85.94 \\
\hline $\begin{array}{l}\text { Carfentrozone ethyl 40\% DF @ } \\
12.5 \mathrm{~g} \mathrm{ml}^{-1} \text { a.i. ha }{ }^{-1} \text { at } 30 \text { DAS }\end{array}$ & 47.67 & 5.33 & 4.16 & 198.89 & 381.24 & 1850.70 & 3812.44 & 78.64 \\
\hline $\begin{array}{l}\text { Carfentrozone ethyl 40\% DF @ } 25 \\
\mathrm{~g} \mathrm{ml}^{-1} \text { a.i. ha }{ }^{-1} \text { at } 30 \text { DAS }\end{array}$ & 37.33 & 5.00 & 7.33 & 267.63 & 504.80 & 2489.30 & 4863.22 & 86.19 \\
\hline $\begin{array}{l}\text { Carfentrozone ethyl 40\% DF @ } 50 \\
\mathrm{~g} \mathrm{ml}^{-1} \text { a.i. ha }{ }^{-1} \text { at } 30 \text { DAS }\end{array}$ & 40.00 & 5.00 & 6.14 & 243.04 & 465.54 & 2259.90 & 4655.39 & 87.32 \\
\hline $\begin{array}{l}\text { Carfentrozone ethyl } 40 \% \text { DF @ } \\
12.5 \mathrm{~g} \mathrm{ml}^{-1} \text { a.i. ha-1 at } 40 \text { DAS }\end{array}$ & 39.67 & 3.67 & 4.11 & 161.73 & 309.72 & 1503.50 & 3097.21 & 81.38 \\
\hline $\begin{array}{l}\text { Carfentrozone ethyl } 40 \% \text { DF @ } 25 \\
\text { g ml }^{-1} \text { a.i. ha }{ }^{-1} \text { at } 40 \text { DAS }\end{array}$ & 39.67 & 4.00 & 4.33 & 171.38 & 328.24 & 1593.40 & 3282.40 & 83.99 \\
\hline $\begin{array}{l}\text { Carfentrozone ethyl } 40 \% \text { DF @ } 50 \\
\mathrm{~g} \mathrm{ml}^{-1} \text { a.i. ha }{ }^{-1} \text { at } 40 \text { DAS }\end{array}$ & 42.00 & 4.33 & 3.93 & 163.40 & 312.91 & 1519.00 & 3020.02 & 86.05 \\
\hline $\begin{array}{l}\text { Carfentrozone ethyl } 40 \% \text { DF @ } 25 \\
\text { g ml- }^{-1} \text { a.i. ha }{ }^{-1} \text { at } 30 \text { DAS (market } \\
\text { product) }\end{array}$ & 39.00 & 5 & 6.46 & 249.66 & 478.31 & 2321.90 & 4602.07 & 81.99 \\
\hline $\begin{array}{l}\text { Oxyflourofen } 23.5 \% \text { EC @ 150-240 } \\
\text { a.i. ha-1 at } 30 \text { DAS }\end{array}$ & 43.33 & 4.67 & 5.34 & 231.92 & 423.84 & 2157.60 & 4238.36 & 79.96 \\
\hline $\begin{array}{l}\text { Bispyribac sodium } 10 \% \text { SC @ } 20 \mathrm{~g} \\
\mathrm{ml}^{-1} \mathrm{ha}^{-1} \text { at } 30 \text { DAS }\end{array}$ & 40.00 & 4.67 & 5.61 & 224.92 & 430.93 & 2091.88 & 4309.27 & 81.75 \\
\hline Untreated control & 44.00 & 4.33 & 3.39 & 149.31 & 286.09 & 1388.80 & 2860.93 & 0.00 \\
\hline Hand weeding at 20 and 40 DAS & 41.00 & 5.33 & 6.71 & 275.00 & 526.85 & 2557.50 & 5268.45 & 85.74 \\
\hline SEm \pm & 2.11 & 0.36 & 0.40 & 16.01 & 30.12 & 148.83 & 278.80 & 0.88 \\
\hline $\operatorname{CD}(p=0.05)$ & NS & NS & 1.10 & 44.38 & 83.48 & 412.53 & 772.80 & 2.43 \\
\hline
\end{tabular}


different treatment at harvest is presented in Table 3. It was observed from Table 3 that weight of $\operatorname{straw}\left(\mathrm{m}^{-2} \mathrm{~g}\right)$, Grain yield and straw yield was influenced significantly due to different weed control treatments.

Treatment hand weeding at 20 and 40 DAS recorded significantly higher straw weight per sq. mt., grain yield and straw yield as compared to rest of treatments under study except application of Carfentrozone ethyl 40\% DF @ $50 \mathrm{~g}$ $\mathrm{ml}^{-1}$ a.i. ha ${ }^{-1}$ and @ $25 \mathrm{~g} \mathrm{ml}^{-1}$ a.i. ha ${ }^{-1}$ at 30 DAS, application of Carfentrozone ethyl 40\% DF @ $25 \mathrm{~g} \mathrm{ml}^{-1}$ a.i. ha ${ }^{-1}$ at 30 DAS (market product) in case of straw weight per sq. mt., grain yield and straw yieldwhich was at par with treatment hand weeding at 20 and 40 DAS. However, treatment hand weeding at 20 and 40 DAS was also at par with application of oxyflorofen 23.5\% EC @150-240 a.i. ha ${ }^{-1}$ at 30 DAS and application of bispyribac sodium 10\% SC @ $20 \mathrm{~g} \mathrm{ml}^{-1} \mathrm{ha}^{-1}$ at 30 DAS.

Amongst the weed control treatment application of Carfentrozone ethyl 40\% DF @ $25 \mathrm{~g} \mathrm{ml}^{-1}$ a.i. ha ${ }^{-1}$ at 30 DAS recorded significantly higher straw weight per sq. mt., grain yield and straw yield as compared to rest of the weed control treatments under study except application ofCarfentrozone ethyl 40\% DF @ $50 \mathrm{~g} \mathrm{ml}^{-1}$ a.i. ha ${ }^{-1}$ at 30 DAS, application of Carfentrozone ethyl 40\% DF @ $25 \mathrm{~g} \mathrm{ml}^{-1}$ a.i. ha ${ }^{-1}$ at 30 DAS (market product), application of oxyflorofen 23.5\% EC @150240 a.i. ha ${ }^{-1}$ at 30 DAS and application of bispyribac sodium $10 \%$ SC @ $20 \mathrm{gm} \mathrm{ml}^{-1} \mathrm{ha}^{-1}$ at 30 DAS which was at par with treatment application of Carfentrozone ethyl 40\% DF @ $25 \mathrm{~g}$ $\mathrm{ml}^{-1}$ a.i. ha ${ }^{-1}$ at 30 DAS. Similar results were reported by Kumari et al. (2018), Kundu et al. (2017), Ramesha et al. (2017) and Pang et al. (2016).

Data regarding weed control efficiency as influenced by different treatment at harvest is presented in Table 3. It was observed from Table 3 that weed control efficiency at harvest was influenced significantly due to different weed control treatments.

Treatment application of Carfentrozone ethyl 40\% DF @ $50 \mathrm{~g} \mathrm{ml}^{-1}$ a.i. ha- ${ }^{-1}$ at 30 DAS recorded significantly more weed control efficiency as compared to rest of treatments under study except treatments application of Carfentrozone ethyl 40\% DF @ $25 \mathrm{~g} \mathrm{ml}^{-1}$ a.i. ha-1 at 30 DAS, application of Carfentrozone ethyl 40\% DF @ $50 \mathrm{~g} \mathrm{ml}^{-1}$ a.i. ha ${ }^{-1}$ at 40 DAS, application of Carfentrozone ethyl 40\% DF @ $50 \mathrm{~g} \mathrm{~m}^{-1}$ a.i. ha ${ }^{-1}$ at 20 DAS and hand weeding at 20 and 40 DAS which was at par with treatment Carfentrozone ethyl 40\% DF @ $50 \mathrm{~g} \mathrm{ml}^{-1}$ a.i. ha ${ }^{-1}$ at 30 DAS.Similar findings were reported by Kumari et al. (2018), Kundu et al. (2017), Ramesha et al. (2017) and Bhullar et al. (2013).

\subsection{Phytotoxic effect}

Application of Carfentrozone ethyl 40\% DF @ $50 \mathrm{~g} \mathrm{ml}^{-1}$ a.i. per at 20, 30 and 40 DAS had showed yellowing of rice seedlings for 3 days after spraying in the range of 1 to $10 \%$. Later on it recovered. Other phytotoxic symptoms like stunting, necrosis, epinasty, hyponasty, etc. was not observed after spraying of Carfentrozone ethyl 40\% DF @ 12.5, 25 and $50 \mathrm{~g} \mathrm{ml}^{-1}$ a.i. ha ${ }^{-1}$ at 20,30 and 40 DAS. Similar findings were reported by Kumari et al. (2018); Zahan et al. (2018); Ramesha et al. (2017).

\section{Conclusion}

For growing Summer rice under upland situation, crop need to be apply with Carfentrozone ethyl 40\% DF @ $25 \mathrm{~g} \mathrm{ml}^{-1}$ a.i. ha ${ }^{-1}$ at 30 DAS to obtain higher grain yield and straw yield with more weed control efficiency. There was no phytotoxicity effect observed in any of the doses of the testing Carfentrozone ethyl 40\% DF in direct seeded rice crop.

\section{References}

Anonymous, 2017. Agricultural Statistics at a Glance 2016, 489.

Food and Agriculture Organization of the United Nations; FAO 2018. http://www.fao.org/faostat/en/\#data/QC

Chauhan, B.S., Johnson, D.E., 2011. Row spacing and weed control timing affects yield of aerobic rice. Field Crops Research 121, 226-231.

Datta, S.K., Khushi, G.S., 2002. Improving rice to meet food and nutrient needs: Biotechnological approaches. Journal of Crop Production, 6229-247.

Hayashi, S., Kamoshita, A., Yamagishi, J., Kotchasatit, A., Jongdee, B., 2007. Genotypic differences in grain yield of transplanted and direct-seeded rainfed lowland rice (Oryza sativa L.) in northeastern Thailand. Field Crops Res 102, 9-21.

Kaur, S., Singh, S., 2015. Bio-efficacy of different herbicides for weed control in direct-seeded rice. Indian Journal of Weed Science 47(2), 106-109.

Kumar, V., Ladha, J.K., 2011. Direct seeded rice: Recent development \& future research needs. Advances in Agronomy 111, 297-413.

Pathak, H., Tewari, A.N., Sankhyan, S., Dubey, D.S., Mina, U., Singh, V.K., Jain, N., Bhatia, A., 2011. Direct-seeded rice:Potential, performance and problems - A review. Current Advances in Agricultural Sciences 3(2), 77-88.

Singh, M., Sriram, C.V., Hanji, M.B., Prabhukumar, S., Kishor, N., 2012. Crop-weed competition and weed management studies in direct-seeded rice (Oryza sativa). Indian Journal of Agronomy 57(1), 38-42.

Lemerle, D., Verbleek, B., Cousens, R.D., Coombes, N.E., 1996. The potential for selecting wheat cultivars strongly competitive against weeds. Weed Research 36, 505-513.

Mahajan, G., Chauhan, B.S., 2011. Effects of planting pattern and cultivar on weed and crop growth in aerobic rice system. Weed Technology 25, 521-525.

Mahajan, G., Brar, L.S., Sardana, V., 2004. Efficacy ofclodinafop against isoproturonresistantPhalarisminorin relation to wheat cultivars and spacing. Indian Journal of Weed Science 36, 166-170.

Bhullar, M., Kaur, S., Kaur, T., Singh, T., Singh, M., Jhala, A., 
2013. Control of broadleaf weeds with post-emergence herbicides in four barley (Hordeum spp.) cultivars. Crop Protection 43, 216-222.

Pang, N., Cui, Y., Hu, J., 2016. Weather dependent dynamics of the herbicides florasulam, carfentrazone-ethyl, fluroxypyr-meptyl and fluroxypyr in wheat fields through field studies and computational simulation, Chemosphere, 165, 320-328.

Singh, V.P., Singh, G., Singh, M., 2004. Effect of fenoxaproppethyl on transplanted rice and associated weeds. Indian Journal of Weed Science 36(3\&4), 190-192.

Verma, S.K., Singh, S.B., Meena, R.N., Prasad, S.K., Meena, R.S., Gaurav, 2015. A review of weed management in India: The need of new directions for sustainable agriculture. The Bioscan 10(1), 253-263.
Ramesha, Y.M., Bhanuvally, M., Gaddi, A.K., Krishamurthy, D., Umesh, M.R., 2017. Efficacy of propanil against weeds in direct seeded rice and its effect on succeding Crop. International Journal of Plant \& Soil Science 17(3), 1-7. Zahan, T., Muktadir, M.A., Rahman, M.M., Ahmed, M.M., 2018. Response of the succeeding crops as affected by the residue of herbicides applied in wheat in Old Brahmaputra Floodplain, Bangladesh, Annals of Agrarian Science (In Press).

Kundu, C.K., Biswas, U., Kundu, A., Bera, P.S., Sathish, G., 2017. Studies on Bio efficacy and Phytotoxicity of Pretilachlor $30.7 \%$ EC in Direct Seeded Rice. International Journal of Chemical Studies 5(5), 1847-1850. 